\title{
Les « bains de sable » dans le Tafilalt (Maroc)
}

Pratiques et représentations de l'immersion des corps en contexte saharien

"Sand baths" in Tafilalt (Morocco)

\section{Marie-Luce Gélard}

\section{(2) OpenEdition}

Journals

Édition électronique

URL : https://journals.openedition.org/tc/7236

DOI : $10.4000 /$ tc. 7236

ISSN : 1952-420X

Éditeur

Éditions de l'EHESS

\section{Édition imprimée}

Date de publication : 15 décembre 2013

Pagination : 100-121

ISBN : 978-2-7351-1654-6

ISSN : 0248-6016

\section{Référence électronique}

Marie-Luce Gélard, « Les « bains de sable » dans le Tafilalt (Maroc) », Techniques \& Culture [En ligne], 61 | 2013, mis en ligne le 15 décembre 2016, consulté le 29 septembre 2022. URL : http:// journals.openedition.org/tc/7236; DOI : https://doi.org/10.4000/tc.7236 


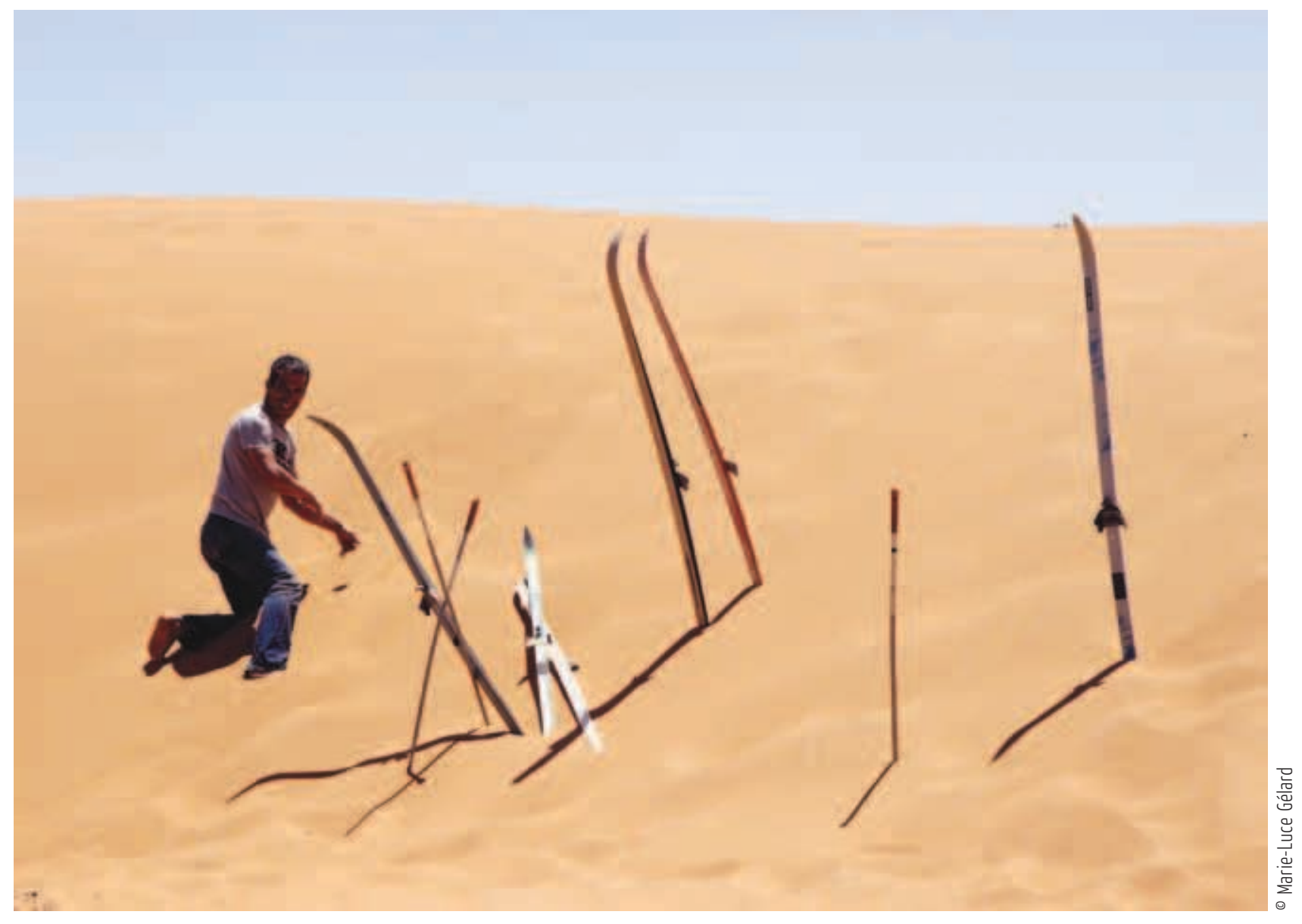




\section{LES « BAINS DE SABLE » DANS LE TAFLLALT}

\section{Pratiques et représentations de l'immersion des corps en contexte saharien}

L'univers saharien engendre de nombreux usages du sable et de la chaleur à des fins pratiques et prophylactiques, dont les «bains de sable » actuellement en pleine expansion sont un bel exemple.

«Sablo-thérapie », « psammatothérapie », « tourisme thérapeutique » et « tourisme de santé » tels sont quelques-uns des nouveaux termes qui décrivent aujourd'hui les bienfaits du sable saharien, principalement au Maroc, en Algérie, en Tunisie et en Égypte. Chauffé par le soleil, le sable contribue, dit-on, à la guérison de nombreuses maladies. Les populations urbaines des régions littorales atlantiques humides et des villes du nord du Maroc soignent leurs douleurs articulaires et particulièrement leurs rhumatismes dans les « bains de sable » qui ont acquis une grande renommée. La région de Merzouga ${ }^{1}$ qui était surtout réputée pour son tourisme de désert (attraction occidentale pour les dunes de sable ${ }^{2}$ ), est désormais connue également de tous les Marocains pour ses étranges immersions dans le sable brûlant durant les mois de juin, juillet et août. Bien que le tourisme de désert soit devenu un objet d'analyses et d'études ethnologiques ${ }^{3}$ (Varichon $\&$ Roccella 2006, Cauvin Verner 2007, Battesti 2009, Picon-Lefebvre \& Chaouni 2012), l'examen de la pratique des «bains de sable " n’a pas donné lieu à des recherches approfondies. Les éléments présentés dans ce texte sont issus de recherches menées dans la région saharienne de Merzouga depuis 1997 et d'enquêtes réalisées en 2008, 2010, 2012 et 2014 notamment à M'hammid, les données algériennes proviennent d'informations fournies par des Algériens vivant dans la région de Taghit et de Timimoun (recueillies en 2010 en Algérie). Au Maroc, le village de Merzouga fortement investi par les curistes permet de mesurer l'impact des « bains de sable » sur plus d'une dizaine d'années. 


\section{Merzouga et région de M'hammid}

Lieux des bains de sable au Maroc carte M.-L. Gélard

Localisation du village de Merzouga et de l'erg Chebbi sur image satellite
Il apparaît utile de se poser la question du vide bibliographique ${ }^{4}$ (Ouajjou 2005) quant aux formes locales de tourisme, mobilités thérapeutiques et/ou récréatives. Issu d'un tourisme national, l'attrait pour les «bains de sable » s'est pourtant accentué au point de devenir, à Merzouga, un enjeu majeur d'une part pour les revenus des villageois et d'autre part pour l'urbanisation massive qui en résulte.

D'où provient ce goût prononcé pour une pratique qui transfigure par l'afflux ${ }^{5} \mathrm{de}$ curistes ${ }^{6}$ les espaces sahariens durant l'été? Quelles représentations du corps et de ses maux déterminent l'usage du sable? Comment expliquer un tel engouement?
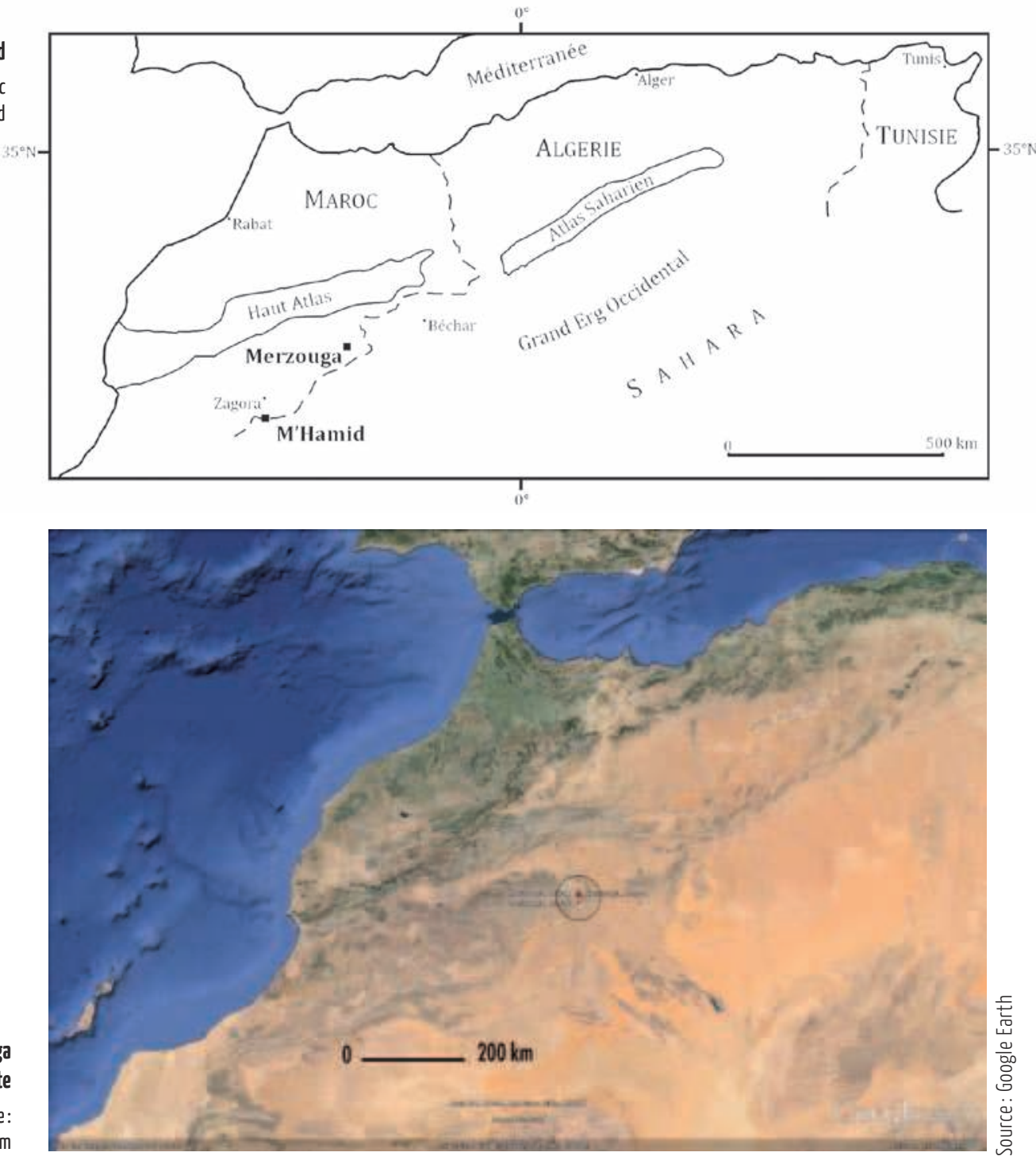

Au Maroc, les « bains de sable » sont attestés dans le sud-est, en Algérie au cœur du grand erg occidental et dans les villes de Ouargla et d'Hassi Messaoud, en Tunisie dans le grand erg oriental et en Égypte dans l'oasis de Siwa. 


\section{L'origine des « bains de sable »: invention ou re-création?}

Au Maroc, la pratique débute à la fin des années 1980 en même temps que l'expansion du tourisme saharien ${ }^{7}$. À l'époque, les seuls hébergements touristiques se faisaient sous la tente, le long de l'erg Chebbi à environ $10 \mathrm{~km}$ du village de Merzouga, avant que n'apparaissent des structures pérennes ${ }^{8}$. Les curistes sont des urbains venant des principales villes du nord du royaume, il s'agit majoritairement d'un tourisme national auxquels s'ajoutent des immigrés établis en Europe. Ils s'installent chez l'habitant dans les villages de Merzouga et d'Hassyblyed, ainsi que dans les hameaux qui jouxtent l'erg.

Lapparition du phénomène au Maroc a lieu simultanément dans le Tafilalt et plus à l'ouest dans la région saharienne de M'hammid au sud de Zagora (cf. carte). Ces deux endroits disposent en effet de formations dunaires de taille significative. Plusieurs interprétations sont données quant à l'origine de ces cures singulières. La première est fournie par les habitants de Merzouga qui auraient inventé la pratique pour attirer des touristes. Les habitants de M'hammid les auraient imités. Dans la région de M'hammid c'est la version inverse qui m’a été donnée. Ces deux espaces sahariens se livrent à une concurrence acharnée pour attirer les visiteurs. À M'hammid, on appâte les touristes en montrant la photographie de la grande dune de Merzouga (Lala Merzouga) pour faire croire que les dunes y sont toutes aussi grandes, alors qu'en réalité l'erg Chegaga (près de M'hammid) est bien moins étendu et ses dunes moins hautes que celles de l'erg Chebbi qui domine Merzouga. Ainsi se joue une quasi-guerre de l'image des sables.

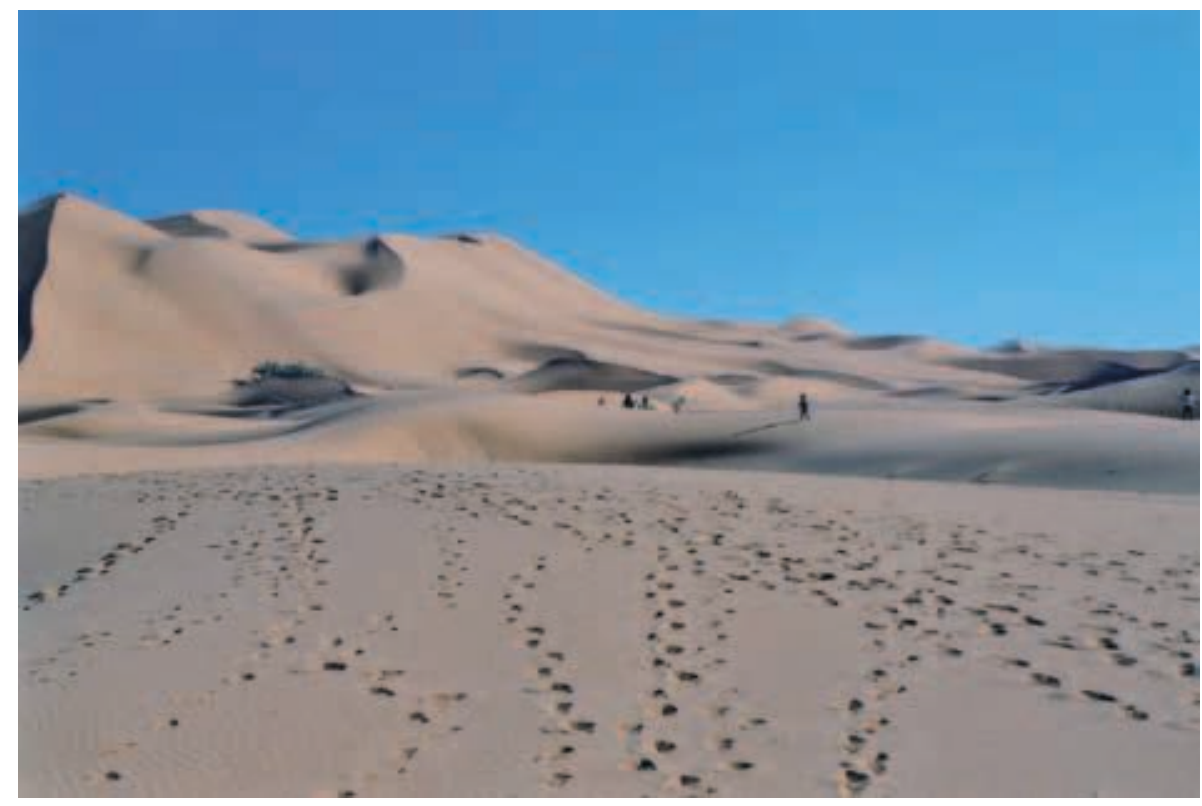

«Lala Merzouga », haute formation dunaire près du village de Merzouga, 2005

La deuxième explication ${ }^{9}$ est d'ordre thérapeutique et anecdotique ${ }^{10}$ : un médecin aurait dit à un immigré souffrant de rhumatismes d'aller se soigner à Merzouga, avec la chaleur du sable. En effet, nombre de Marocains vivant en Europe disent souffrir du 
froid et de l'humidité, agents responsables de rhumatismes articulaires mais aussi de paralysies des membres inférieurs, de polyarthrite ou de lombalgie.

Les modalités d'application de cette thérapeutique se seraient inspirées, rapportent les Ait Khebbach, des techniques anciennes utilisées pour réparer les fractures osseuses. Après réduction si nécessaire de la fracture, les vieux conseillaient de maintenir la partie du corps blessée sous le sable chaud. Autrefois, les nomades chauffaient le sable avec des braises et s'y allongeaient afin de soulager leurs douleurs lombaires. Les nomades qui souffraient dit-on fréquemment du froid, associaient le feu et le sable pour lutter contre lui. Mal vêtus, ayant enduré toute leur vie des hivers froids, « les vieux pour ne pas souffrir des jambes s'enterraient dans le sable ». La technique et l'usage auraient donc une origine ancestrale... L'institutionnalisation de la pratique, devenue « bains de sable » s'enracinerait donc pour la plupart des Ait Khebbach, dans les usages des « Anciens ». Le mode de vie autrefois nomade des Ait Khebbach est l'objet d'une valorisation qui n'est pas simplement liée à la nostalgie de leur puissance et de leur autonomie passées mais trouve un ancrage identitaire plus profond. En témoignent différents rituels où les références matérielles ou symboliques au nomadisme sont fréquentes, tels les rituels du mariage et la présence physique de la tente ${ }^{11}$ (Gélard 2008), y compris en milieu urbain.

Le sable chaud est aussi utilisé dans d'autres cas, comme par exemple pour soulager les contractions avant l'accouchement, la parturiente est allongée sur du sable chauffé, le plus souvent dans une poêle posée sur le gaz. D’autres médications instrumentalisent la chaleur à des fins thérapeutiques. L'empreinte environnementale sur les pratiques corporelles est conséquente. Ainsi, les principales caractéristiques climatiques, aridité et chaleur, sont pensées comme salvatrices et bénéfiques. De fait, on assiste à une élévation des vertus dessiccatives: le sec et le chaud, deux normes du bien-être. Ces éléments sont indispensables à la compréhension des pratiques car la dessiccation est une qualité très appréciée des Sahariens.

La pratique des bains de sable illustre comment dans l'univers saharien, le sec plus précisément l'absence complète d'humidité — sert de référence sensorielle la plus appréciée, dans le traitement du corps malade. Les choix sensoriels s'enracinent dans des cadres géographiques, où l'inscription territoriale des corps renvoie aux questionnements plus vastes et notamment à la nécessité d'une étude des corps dans leur action/ mouvement (J.-P. Warnier: 1999 et 2009).

La dessiccation et la chaleur sont des remèdes à presque tous les maux du corps: brûlures au soufre ou au fer rouge, fumigations, ingestions de produits desséchés, etc. Ce sont essentiellement les maux invisibles du corps, ces désordres intérieurs qui nécessitent réchauffement et échauffement. Il convient aussi d'extraire du corps l'humidité et l'eau considérées comme responsables des désordres. La dessiccation renvoie à l'opération par laquelle on prive un élément de tout ou partie de l'humidité qu'il renferme. Dans la médecine allopathique occidentale, les médicaments dessiccatifs désignent ceux usités pour absorber les sérosités (ou le pus d'une plaie par exemple), alors qu'ici la propriété dessicative s'applique spécifiquement au milieu corporel interne.

Lors d'une faiblesse générale (perte d'appétit et de poids) il faut « chauffer le corps ». Aussi, à l'aide d'une tige de fer portée au feu, on pratique des brûlures en stries parallèles sur la peau, distantes de quelques centimètres, réalisées le plus souvent le long de la colonne vertébrale ou sur les bras et les épaules. Les brûlures sont superficielles, la chaleur ainsi diffusée dans le corps doit lui rendre toute sa vigueur. D'autres brûlures faites sur des parties malades du corps (ventre, dos et articulations) à l'aide de soufre (lchbrite) sont aussi communes. Pour ce faire, on dépose sur la partie du corps malade une pincée de soufre que l'on enflamme. Le 
soufre se consume lentement puis s'éteint, la douleur est très vive et perdure, car la brûlure de la peau est profonde. Nombre d'habitants de Merzouga et de la région ont des marques rondes spécifiques de cette thérapeutique. La cicatrisation est lente et participe de l'efficacité du traitement. Il faut éviter que la blessure ne se referme trop vite, car c'est par cette ouverture du corps que le mal ${ }^{12}$ trouve une porte de sortie. Il en est de même des crevasses aux pieds, qui ne sont pas soignées afin de laisser cette issue naturelle à la sortie du mal. À défaut ce dernier serait susceptible de créer des dégâts irréversibles à l'intérieur du corps.

C'est dans ce contexte des perceptions des désordres intérieurs du corps associées à une valorisation environnementale de l'espace saharien que les « bains de sable » trouvent leur origine et leur développement contemporain. Le désert est pensé comme le lieu par excellence de la guérison des corps meurtris par la vie urbaine (humidité des villes du littoral, pollution, etc.) d'où l'origine géographique des curistes.

Durant près de 4 mois, le village de Merzouga est littéralement transformé. Le tourisme de désert modifie peu la physionomie villageoise du fait de son implantation dans des structures hôtelières extérieures au village (à plusieurs kilomètres au nord). Par contre l'arrivée des curistes engendre des modifications démographiques de taille. En 2010 et 2014, on ne trouvait plus une chambre disponible et on relevait la présence de trente bouchers à Merzouga contre trois en morte-saison. Deux médecins généralistes avaient été envoyés sur place, le dispensaire du village ne disposant que de trois infirmiers d'État. On compte plusieurs dizaines d'herboristes ${ }^{13}$ qui s'installent provisoirement et près d'une vingtaine de restaurants temporaires. Les marchands de légumes sont tout aussi nombreux et fleurissent d'autres commerces ambulants: vendeurs d'orangeade, de crêpes, de beignets, etc. Tout le monde peut s'improviser commerçant saisonnier et proposer des produits, alimentaires ou autres. Ce commerce prospère fournit des revenus substantiels aux habitants de Merzouga et de la région. La fréquentation est telle dans le quartier du souk que la circulation est modifiée, un sens unique imposé, contraignant les curistes qui circulent en véhicule à effectuer le tour complet du quartier pour s'approvisionner.

\section{En pratique}

Les « bains de sable » sont réalisés aux heures les plus chaudes de la journée entre 11 heures et 13 heures ${ }^{14}$. Ils consistent en une immersion du corps dans le sable brûlant (70 à $80^{\circ} \mathrm{C}$ ) lequel est censé absorber l'excédent d'humidité contenu dans l'organisme, plus particulièrement dans les os. Après avoir aménagé dans le sable une petite dépression, l'accompagnateur/aide du patient l'invite à s'y allonger et le recouvre de sable à l'exception du visage et de la poitrine. Il est donc nécessaire d'être secondé. Les femmes le sont par des jeunes filles et les hommes par des jeunes hommes ou garçons du village. Ces aides sont rémunérés selon des tarifs variant entre 7,5 et 25 dirhams (dh) la « séance ». Celle-ci comprend l'accompagnement dans un lieu propice, il convient de trouver une petite dune, une surface en légère pente est nécessaire car elle permet de s'allonger la tête légèrement surélevée. Il faut ensuite aménager un « trou » de la taille du corps puis recouvrir le patient de sable. Enfin, l'assistant dépose un tissu de protection sur le visage pour le protéger du soleil et demeure à côté du curiste durant toute la durée du bain. 
Hameau de Taqucht, juillet 2008, $13 \mathrm{~h}$. Température extérieure $44^{\circ} \mathrm{C}$.

On ne peut marcher sur le sable brûlant. L'assistante de la curiste porte la pelle destinée à creuser le sable. Elle est rémunérée au nombre de « trous » effectués Le « trou » comprend le creusement, le recouvrement et la surveillance.

Le choix du lieu dépend des connaissances de l'assistante: dune en pente légère, sable propre et exposition plein sud.

Creusement du « trou » par l'assistante. La température est élevée contraignant à protéger le visage (chèche et port de lunettes: ici un masque de ski). Objets « exotiques » laissés par les touristes ${ }^{15}$

La curiste sallonge dans le « trou » et retire ses vêtements. Le contact de la peau avec le sable est indispensable à l'efficacité curative.

De la graisse est apposée au niveau des articulations.

La curiste est progressivement recouverte de sable déposé sur son corps.

Les femmes conservent leur chemise mais la soulèvent pour que le ventre soit au contact du sable. La poitrine est toujours protégée.
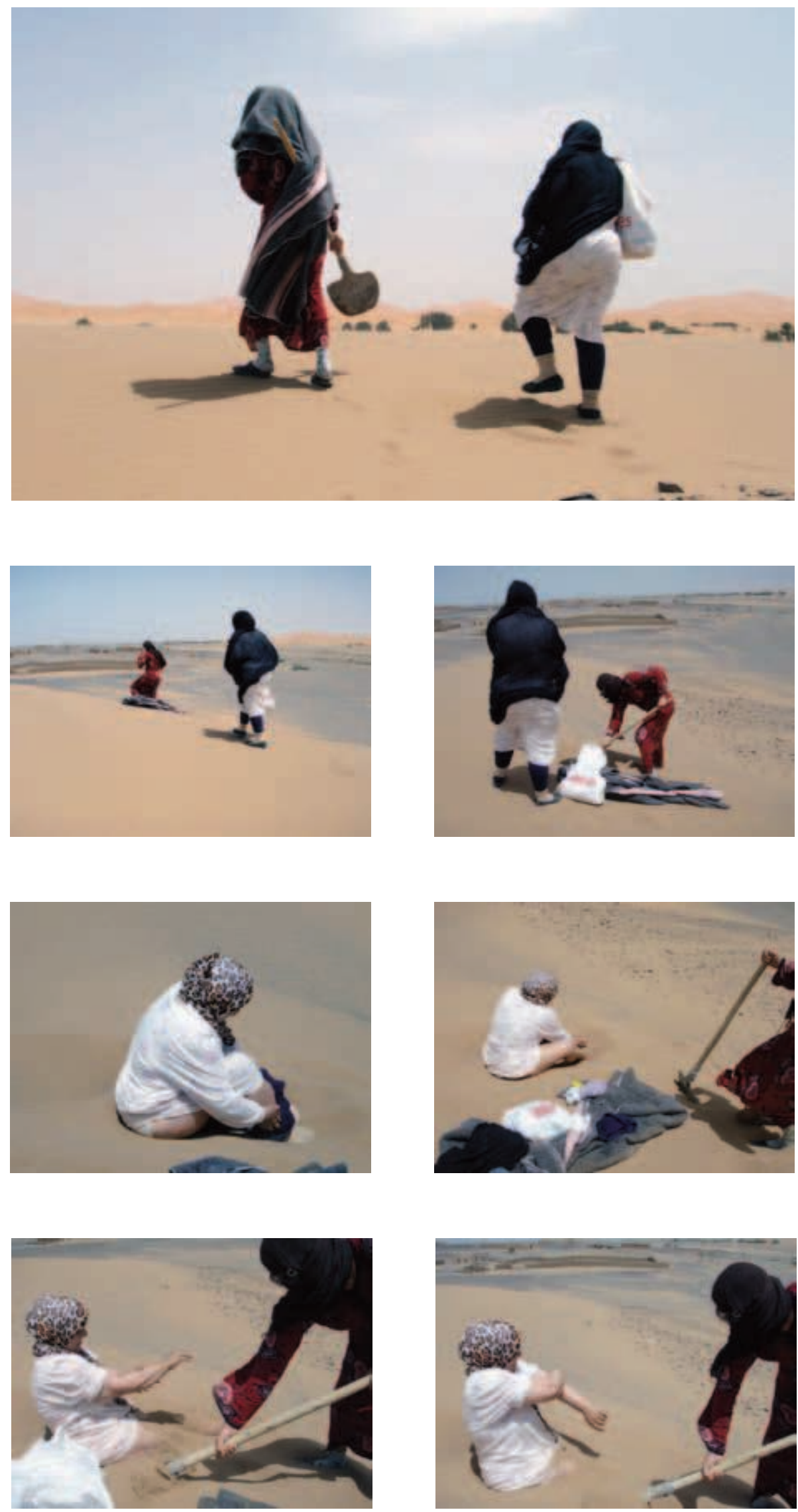

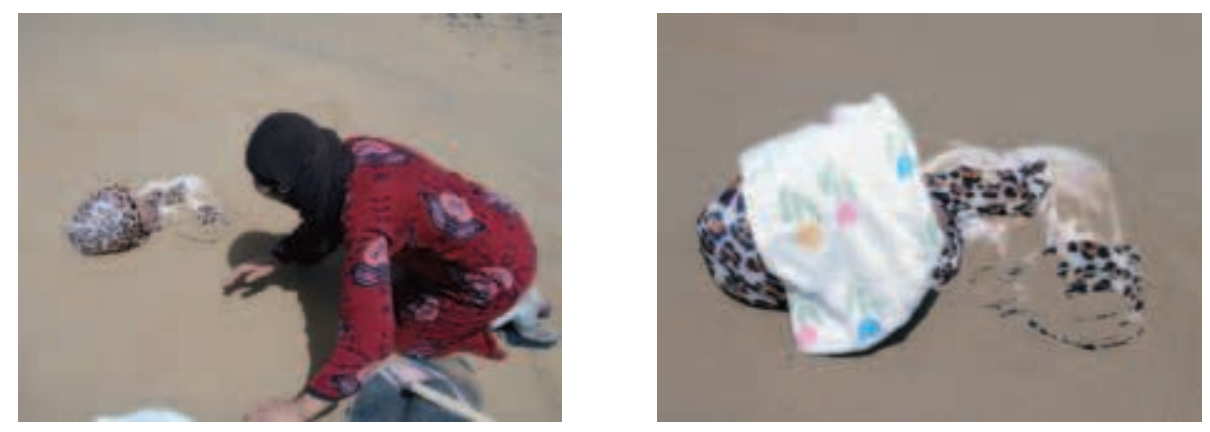

On dépose à la main le sable sur la poitrine en évitant de le déposer sur le cœur.

Immersion du corps durant 20 minutes. Le visage est protégé des rayons du soleil.
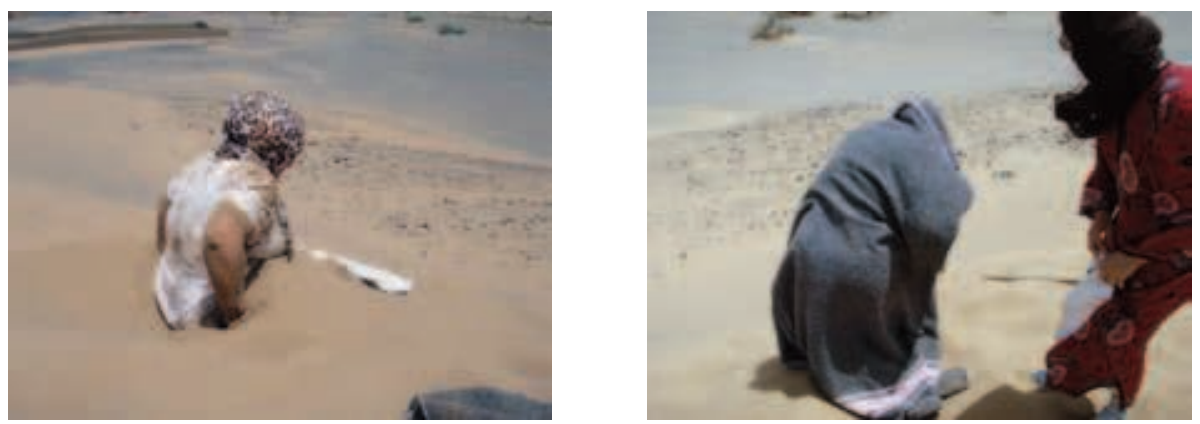

La patiente s'extrait seule du sable mouillé par la sueur.

À peine sorti du sable, le corps doit être protégé de la différence thermique entre l'intérieur du sable ( $70^{\circ} \mathrm{C}$ environ) et l'air ambiant $\left(44^{\circ} \mathrm{C}\right)$ et protégé du vent, très fréquent en été qui augmente le " choc thermique ».

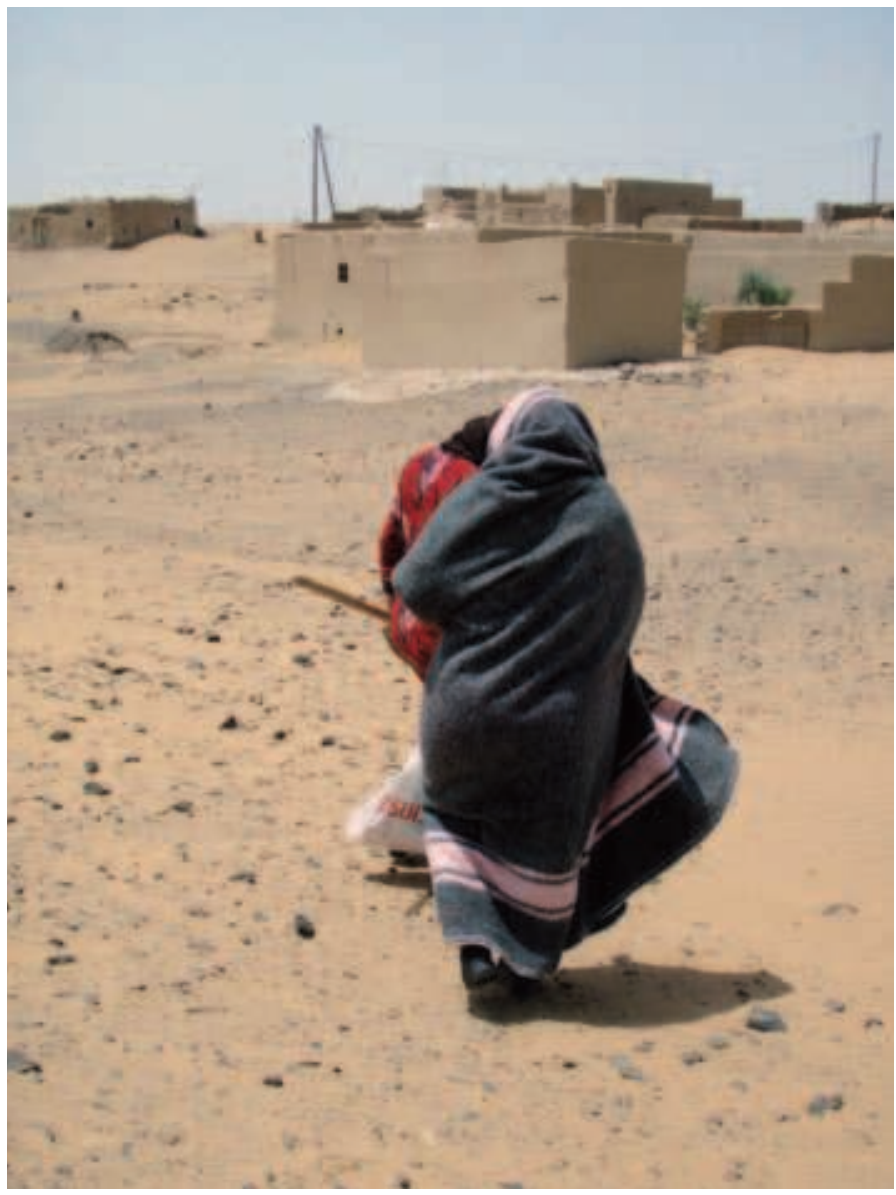

Retour à l'abri dans une chambre chez l'habitant. La patiente ne devra boire que du thé durant plusieurs heures, avant d'être autorisée à boire de l'eau ${ }^{16}$. 
Le curiste demeure dans le sable pendant 10 à 40 minutes, puis se dégage. Les durées sont variables et dépendent de la volonté du patient et des conseils qui lui ont été prodigués. Depuis le début des années 2000, compte tenu de la fréquence des accidents cardiovasculaires mortels, la réduction des temps d'exposition s'est généralisée. Il est aussi nécessaire pour les curistes les plus âgés de consulter au préalable un médecin généraliste, ou un infirmier du dispensaire.

«L'infirmier prend la tension et dit oui ou non. Il te donne aussi une estimation de la durée. C'est important car il y a eu beaucoup de morts » (Ahmed, décembre 2011).

Au contact du corps, le sable est imbibé de sueur parfois sur une dizaine de centimètres d'épaisseur. "Tu sens le sable qui aspire le froid en toi. Il faut le faire 10 à 15 fois 15 minutes environ» (Ahmed, décembre 2011).

« On peut aller jusqu'à une demi-heure selon le ressenti car tu sens la circulation sanguine qui s’accélère » (Moha, avril 2013). «Une fois que tu es enterré, dans les 5 minutes tu sens la sueur et tu entends le cœur qui bat » (Ahmed, décembre 2011).

Il arrive que durant la séance, l'on ajoute du sable sec sur les parties qui se trempent de sueur. C'est le cas lorsqu'on ne dépose qu'une faible quantité de sable sur le corps.

Dégagé du sable, le curiste s'enroule dans une couverture et demeure quelques heures au repos. Il ne peut boire d'eau fraîche et seuls quelques verres de thé sont permis. Depuis quelques années, les discours ont changé, l'interdiction de boire est devenue moins stricte, certains prétendant même qu'il est indispensable de boire abondamment pour éviter une déshydratation.

Durant le bain, c'est la circulation de l'air entre les grains de sable agissant comme un isolant thermique qui permet de supporter des températures très élevées. C'est dit-on la température et le poids du sable conjugués qui entraînent une augmentation de la pression artérielle et créent une chaleur qui ne cause pas de brûlures. Selon le discours des praticiens, le contact du sable chaud entraîne une meilleure circulation sanguine, débarrasse d'un poids, désintoxique le corps, etc.

En Algérie, dans la ville saharienne de Taghit ${ }^{17}$ à quelques kilomètres à l'est de Merzouga, on observe un phénomène d'expansion des «bains de sable » très semblable à celui de la région. À Taghit, on met aussi en avant les vertus curatives des « bains de sable » pour les maladies de peau grâce aux sels et aux composants organiques présents dans le sable.

Le sable devient une sorte de substitut à l'eau et la comparaison est d'autant plus pertinente si on se réfère au traditionnel bain maure (bain de vapeur autrement appelé hammam au Maghreb, dont l'usage est collectif). Celui-ci n'existe pas à Merzouga, par contre les femmes réalisent un bain de vapeur individuel, très économe en eau. Il consiste à rester sous une bâche en plastique suspendue à un trépied de bois, au centre et sous laquelle une bassine est remplie de quelques litres d'eau bouillante. Un tabouret est posé dans la bassine sur lequel on s'assoit. La vapeur s'échappe de l'eau et la bâche en plastique disposée en plein soleil entretient la production de chaleur. Le savonnage et la friction de la peau pour en éliminer les impuretés sont pratiqués comme dans un hammam plus classique, mais de manière strictement individuelle ${ }^{18}$ (Gélard 2014). 


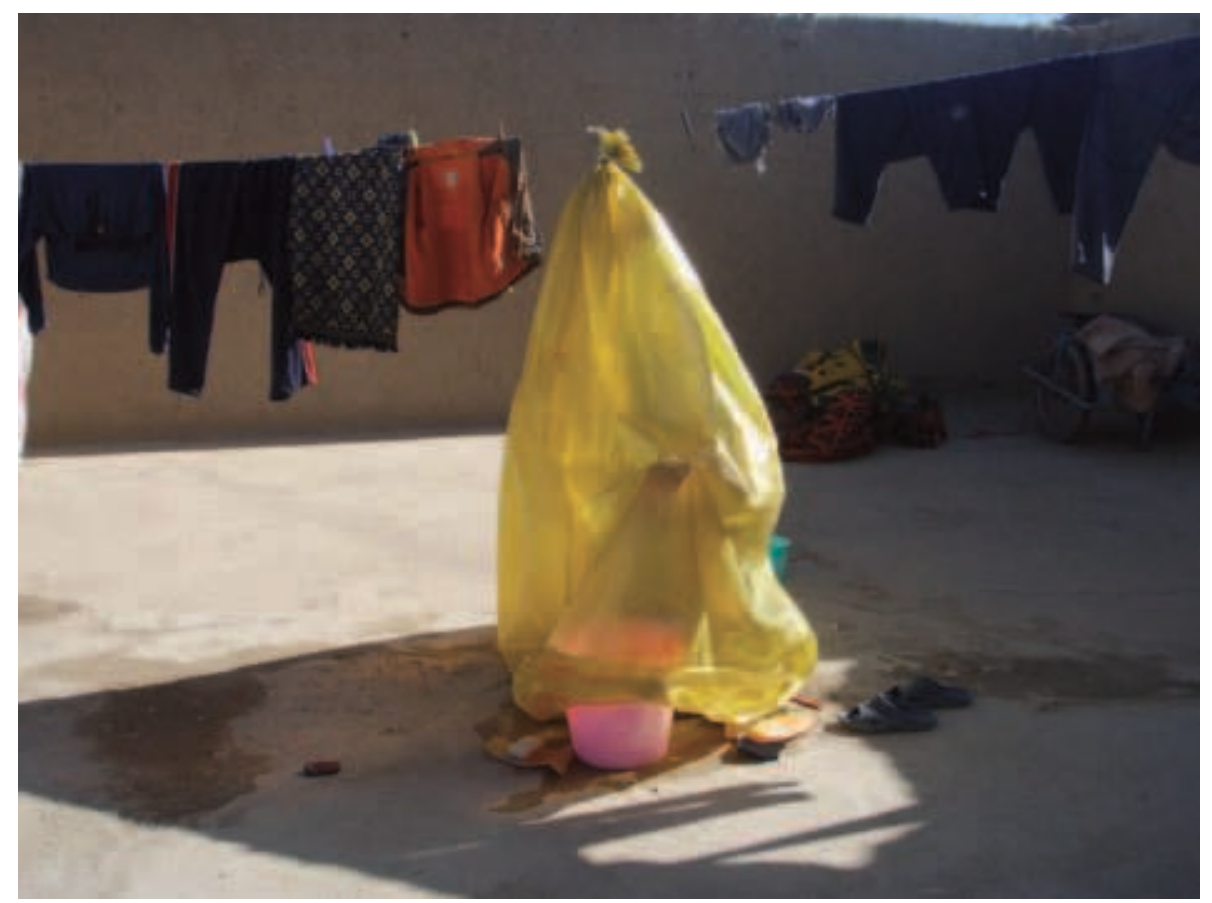

Bain de vapeur.

On distingue en transparence

un pied. La chaleur sous la bâche est très forte.

Dispositif installé sous la bâche. C'est la vapeur et la chaleur qui sont utilisées avec quelques litres d'eau seulement, 2010

\section{« Bains de sable » et tourisme}

Divers sites d'infrastructures touristiques de Merzouga publicisent les « bains de sable »; ils évoquent la « santé au désert » via la « sabulum-thérapie ${ }^{19}$ », « la psammatothérapie », « l'arénothérapie » qui met à profit les propriétés absorbantes du sable et la chaleur du soleil.

Ces nouveaux vocables employés sont récents, ils s'adressent à une clientèle européenne, l'immersion curative dans le sable ayant peu d'adeptes encore auprès des touristes occidentaux. Il convient de les attirer en utilisant un discours « vendeur », les « bains de sable » sont une « médecine douce » peut-on lire sur les sites qui tentent de s'ouvrir à une clientèle étrangère. Mais on lit aussi quelques lignes plus bas qu'il est indispensable d'aller voir le médecin car « le "bain de sable" n'est pas à prendre à la légère »!

C'est sous la forme d'un échange direct entre sable et corps que la thérapie est explicitée par les praticiens ${ }^{20}$. Les rayons infrarouges seraient emmagasinés dans le sable et restitués au corps par immersion bien au-delà de la peau. On voit comment dans les discours, le glissement entre thérapie destinée au soin des douleurs lombaires ou osseuses peut aussi s’appliquer à des vertus commercialement plus prisées par un public occidental. Ainsi, les rayons infrarouges vont désintoxiquer le corps et explique-t-on « faire fondre la graisse ». Les arguments de vente de la pratique des « bains de sable » sont nombreux et changent au gré des modes. Ainsi, il y a quelques années, on parlait de l'importance, après la cure, d'un massage à la graisse de dromadaire! Aujourd'hui, les Merzougui qui veulent diversifier l'offre touristique avec l'argument des bienfaits du sable, font le parallèle avec l'usage du sable dans les thalassothérapies en Occident où l'on couvre le corps de sable chauffé à $45^{\circ} \mathrm{C}$. On évoque alors un « soin » en parlant de 
l'ensevelissement dans le sable, qui dure une dizaine de minutes. Voici la description que l'on peut lire sur un site français de thalassothérapie et de balnéothérapie (http://www.ligne-balneo.com/ bains-de-sable-et-climatotherapie-marine.html): « La psammatothérapie peut être considérée comme une thérapie exogène à chaleur sèche qui utilise le sable auquel s'ajoute la composante pharmacologique spécifique de l'eau de mer sous forme de sels fixés aux granulés. Le sable a une composition chimique particulière et essentiellement constitué de granulés parmi lesquels est interposé de l'air (système poreux), en représentant d'une telle manière un système à basse conductivité thermique et à superficie spécifique limitéecapable de transférer la chaleur, reçue par le rayonnement solaire, sans entraîner de brûlures malgré le haut degré thermique ».

Plus intéressantes encore sont les indications thérapeutiques des thalassothérapies qui sont identiques à celles des « bains de sable » sahariens: ostéo arthroses, issues et séquelles de formes traumatiques (fractures, distorsions, etc.), rhumatismes extra-articulaires, rhumatismes inflammatoires chroniques, etc. Cependant on explique que c'est l'influence du climat marin (pour les thalassothérapies) qui entre aussi dans les vertus des bains de sable. Il serait utile de proposer une comparaison entre les deux pratiques thérapeutiques. L'adaptation du vocabulaire à une offre touristique naissante est récente mais significative. La diminution notable de la fréquentation touristique due aux circonstances économiques (crise de 2008) et politiques (les «printemps arabes ${ }^{21}$ ) contraint à une diversification maximale de l'offre. Cependant, on constate un engouement très faible du public occidental pour les « bains de sable», principalement en raison du climat trop agressif à son goût.

\section{La désignation des curistes, ces « étrangers » de l'intérieur}

Les habitants de Merzouga emploient plusieurs termes imazighen ${ }^{22}$ pour désigner les curistes, ces appellations sont quelque peu dévalorisantes, elles sont censées ne pas être comprises des amateurs de « bains de sable ». En effet, la grande majorité des curistes sont arabophones et si certains comprennent quelques mots de tamazight (langue berbère) ${ }^{23}$, la langue de communication est l'arabe.

La terminologie vernaculaire la plus courante employée

Scincus scincus (Linnaeus, 1758)

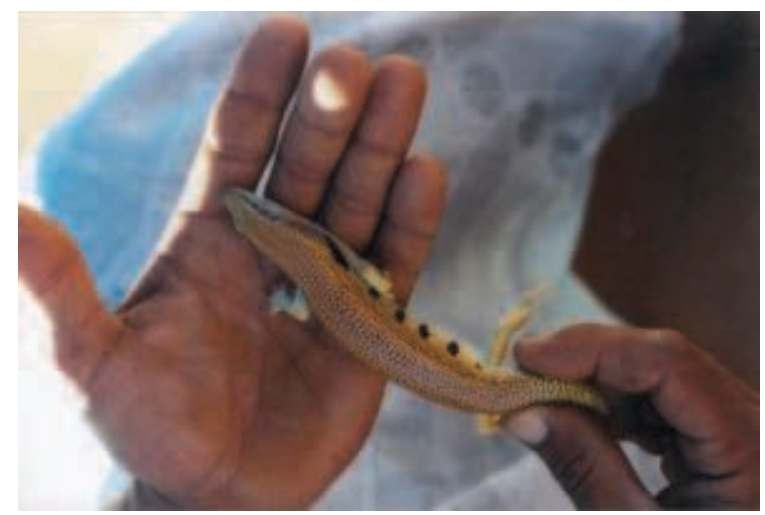
est celle de "poisson de sable » (islman n-talght), en référence au Sincus sincus, sorte de lézard qui se déplace dans le sable à la façon d'un poisson.

La comparaison animalière choisie n'est pas flatteuse, elle est utilisée avec ironie voire raillerie par les Merzougui. L'immersion dans le sable et le dégagement du curiste rappelle la sortie du lézard des sables ${ }^{24}$. L'appellation est aujourd'hui connue de la plupart des curistes interrogés aussi on utilise plus volontiers le mot chghchman qui est le terme amazigh pour désigner le Scincus scincus sans être la traduction littérale de l'euphémisme « poisson de sable » ${ }^{25}$ (Champault 2003). Toujours sur le mode de la moquerie ${ }^{26}$, l'expression amazigh ait ouzgal « ceux qui s'enterrent » est aussi utilisée soulignant l'ironie d'une pratique comparée à un « auto-enterrement ». 

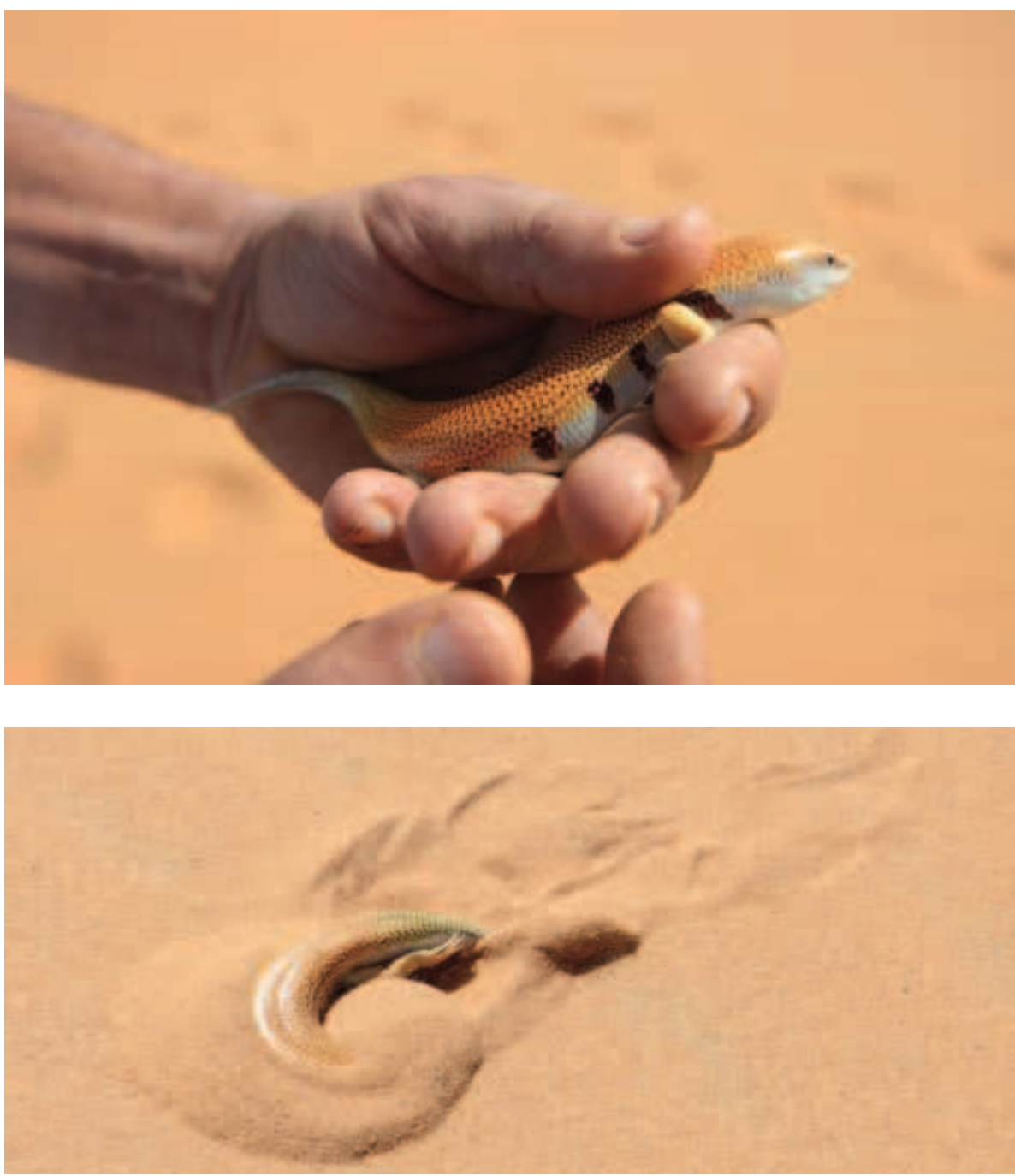

La région de Merzouga est devenue l'un des hauts lieux du tourisme saharien de masse. Le désenclavement de la région par la construction d'une route asphaltée, en 2005, et l'arrivée de l'eau dès 2003, ont contribué à une massification des pratiques touristiques. En quelques années, le tourisme de masse occidental s'est développé autour des villages de Hassylbyed et de Merzouga, mais ces derniers sont peu investis (hormis les quelques commerces des quartiers du souk) du fait d'une implantation des auberges ${ }^{27}$ le long de la formation dunaire (Erg Chebbi).

D'une manière générale, les responsables des auberges ne louent pas aux curistes. Ils soulignent qu'il est difficile de faire cohabiter curistes et touristes occidentaux. Même si à cette saison les seconds sont peu nombreux en raison des températures trop élevées, les premiers « débarquent dans leurs couvertures au bord de la piscine. Ils déposent du sable partout, dans les chambres, sur les lits et bouchent les douches... ${ }^{28}$.

Au final, les curistes vont plutôt être hébergés au sein des villages, chez l'habitant ou dans des maisons construites spécialement à cet effet.
On distingue ses écailles similaires à celle d'un poisson. Merzouga, 1998.

\section{Erg Chebbi}

L'animal « nage » sous la surface du sable. Pour ce faire, il cesse d'utiliser ses membres. Dès qu'il entre dans le sable, son mode de locomotion se modifie totalement. II ondule alors comme une anguille. II utilise toutes les forces de friction, d'une part entre les grains de sable et d'autre part entre le sable et sa peau pour réaliser sa trajectoire. Les descriptions des naturalistes font systématiquement le parallèle entre eau et sable. Le Scincus scincus aime le sable où il vit « comme un poisson dans l'eau ». On le nomme également « poisson du désert ». II vit dans l'ensemble du Sahara dans les grandes zones sableuses. II peut plonger dans le sable grâce à des écailles qui protègent ses yeux et ses oreilles. 2013 
Dans les années 1990, la pratique des « bains de sable » était déjà fort développée mais seul l'un des trois quartiers du village de Merzouga offrait des locations de chambres. Ce tourisme de cure créait des perturbations sociales importantes, car à l'époque où le village ne disposait pas de l'eau courante, les hommes (curistes) allaient chercher l'eau à la source, activité exclusivement féminine. Les femmes et jeunes filles du village les laissaient se servir sans qu'ils attendent leur tour ou même remplissaient leurs bidons afin d'accélérer leur passage. En raison des températures estivales les corvées d'eau s'étaient multipliées et avec elles, la présence gênante des curistes ${ }^{29}$. Le fait que ces derniers soient des citadins augmentait les causes de conflits. Ainsi, en 1998, le Moghazni ${ }^{30}$ dut faire appliquer l'interdiction du port du short par les curistes dans les rues du village. Aujourd'hui, quelques conflits perdurent, mais la fermeture des maisons (murs d'enceinte et portails) permet aux curistes de demeurer à l'abri des regards. En outre les nombreuses modifications architecturales des habitations ont permis de créer des espaces évitant tout contact entre curistes et loueurs. Aujourd'hui, la grande majorité des habitants louent une partie de leur demeure ou une habitation créée spécialement pour les curistes. De nombreuses familles ont construit des maisonnettes dans leur cour intérieure, juste deux ou trois chambres de 2 mètres sur 3, une petite cuisine et un espace toilette. Les accès sont distincts de ceux de l'habitation principale évitant ainsi toute rencontre fortuite entre les curistes et la famille. En l'espace d'une quinzaine d'années, les locations sont devenues courantes et ne sont plus l'objet d'une stigmatisation, du type: «Jamais je vais accepter ça. Des étrangers qui vont rentrer dans ma maison et on va se croiser? Ah ça non! C'est impossible. La maison, c'est sacré, c'est pour moi et c'est pas à louer » (propos recueillis en 1997 à Merzouga). Bien au contraire, nombreux sont les villageois qui construisent des habitations sur leurs terres afin de multiplier leurs revenus. Les

\footnotetext{
Modèles des couvertures dites « des prisonniers » dans lesquelles les curistes s'enveloppent après le « bain de sable » Merzouga, 2008.
}

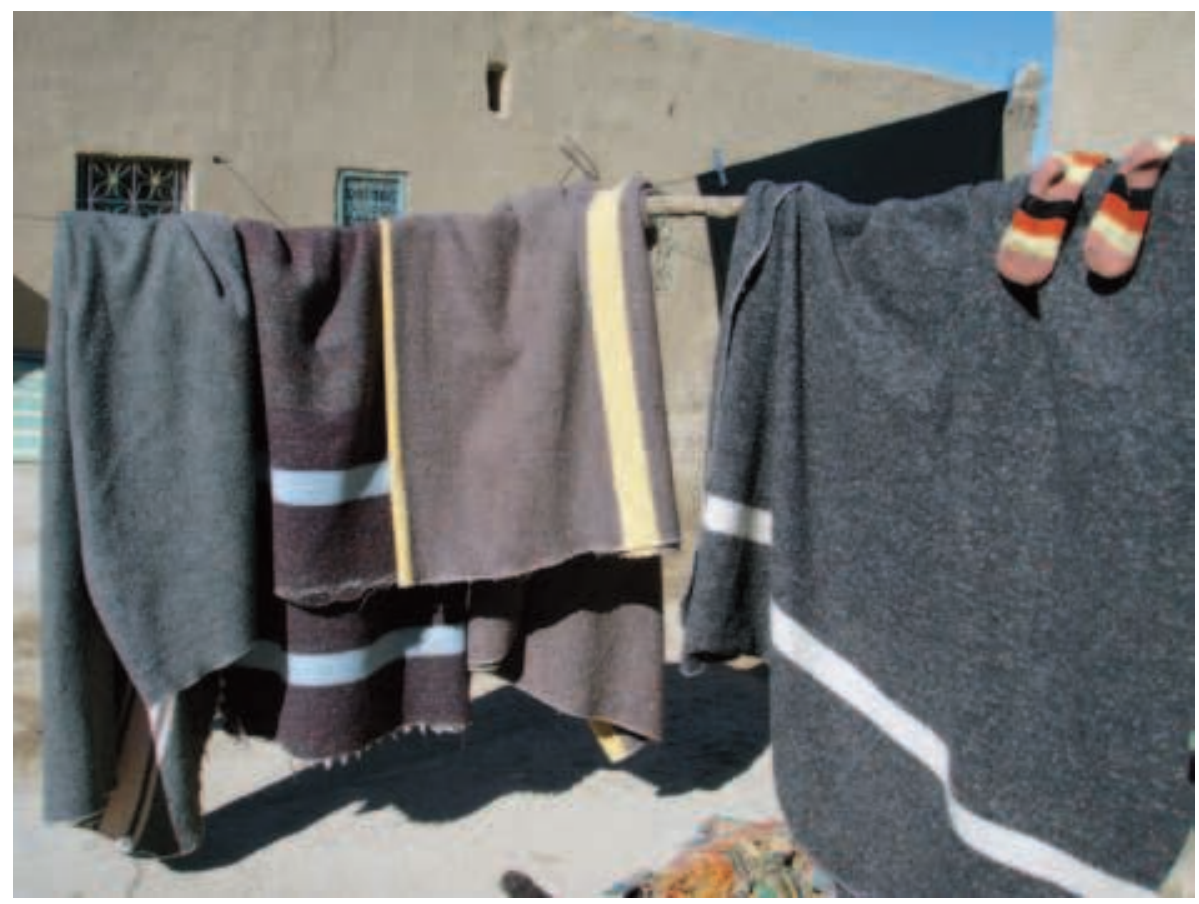




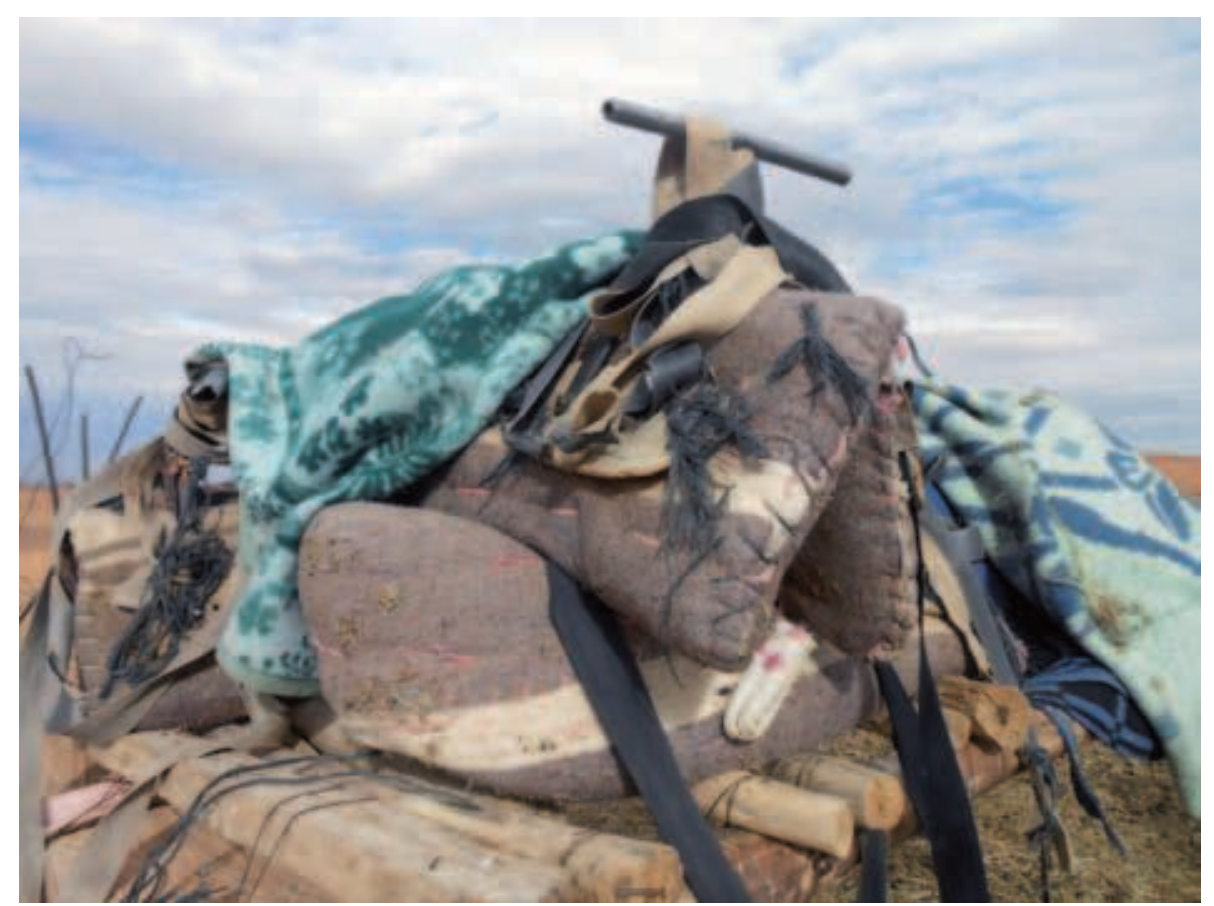

constructions sont très denses et ajoutent à l'urbanisation de l'espace saharien. On souligne même des phénomènes de conurbanisation, à petite échelle comme c'est le cas entre le village de Merzouga et le hameau de Taqucht (autrefois distants de 1,5 km). La sédentarisation des nomades n'est pas la seule cause de cette urbanisation massive, les «bains de sable » jouent un rôle de premier plan dans les constructions d'habitations ${ }^{31}$ (Belguidoum 2002, Pliez 2003, Kouzmine 2012). Le phénomène se ralentit en raison du glissement du Ramadan ${ }^{32}$, période durant laquelle la pratique des « bains de sable » est notablement diminuée.

En 2011, on louait une chambre $50 \mathrm{dh}^{33}$ la nuit avec un accès à une salle de bain et à une cuisine communes. Les locations qui s'échelonnent sur environ quatre mois sont une véritable manne financière pour tous les habitants de la région. Les tarifs varient en fonction des conditions d'hébergement mais il est facile de créer un logement à moindres frais puisqu'on peut louer une pièce avec juste une natte en plastique sur le sol, les couvertures sont achetées par les curistes. Là encore l'appellation est révélatrice, on les nomme les « couvertures des prisonniers». Il s'agit de couvertures en coton, de qualité médiocre ${ }^{34}$ utilisées pour la confection des coussins de bât des dromadaires (bourrelets à coeur de paille qui sont en appui sur l'échine de l'animal).

La nomination des curistes et la dépréciation dont ils sont l'objet sont intéressantes à souligner au regard de l'apport financier important qu'ils représentent. À ce jour, la majorité des habitants de la région bénéficie des retombées financières mais dans l'entre soi villageois, on continue à dévaloriser les « chechman », figure de l'étranger, qui n'entend rien au mode de vie saharien et qui méprise souvent les habitants « du bled ».
Coussins de paille sur lequel repose l'armature en bois et fer de la selle de dromadaire destinée à l'usage touristique

Les couvertures de couleur marron et blanche sont les mêmes que celles utilisées par les curistes lors des « bains de sable ». Erg Chebbi, 2012 


\section{Immersion des corps et immersion des nourritures: la dessiccation salvatrice}

Le climat saharien n'est pas vécu comme néfaste, s'il peut être parfois considéré comme désagréable, la chaleur et ses vertus dessiccatives sont pensées comme salvatrices, elles sont pour les Ait Khebbach les canons du bien être. Un bien être du corps qui transite par les bienfaits de la chaleur. C'est précisément face aux changements introduits récemment comme la conservation des denrées alimentaires (appareils frigorifiques) ou les appareils de climatisation que les réactions sont souvent des rejets purs et simples ou des « réinventions » d'attitudes passées empruntées au « monde nomade ». Le plongeon des corps dans le sable brûlant n'est pas nécessaire aux Sahariens, qui utilisent cependant les vertus du sable par le biais de l'immersion de nourriture dans le sable. C'est la cuisson du pain dans le sable qui présente l'exemple le plus frappant des bienfaits attribués à l'environnement saharien et précisément ici au sable.

On remarque une polarisation de la pratique utilisant deux rituels, l'un pratiqué par les femmes en l'honneur d'une sainte, Lala Merzouga, personnifiée dans la grande dune du village et l'autre par les hommes qui réactivent une pratique pour les besoins $\mathrm{du}$ « folklore touristique .

La cuisson du pain dans le sable est réalisée pour des raisons prophylactiques et/ou religieuses. Lala Merzouga protège le village et lui dispense ses vertus. Accompagnées d'enfants, les femmes s'y rendent pour faire exaucer des voux divers, demander une protection, une guérison, ou le retour d'un proche. Elles partent en groupe, le plus souvent en début d'après-midi. Pour rejoindre Lala Merzouga, il faut une bonne heure de marche dans le sable. Leur station se situe au pied de la dune, près d'un bosquet de tamaris.

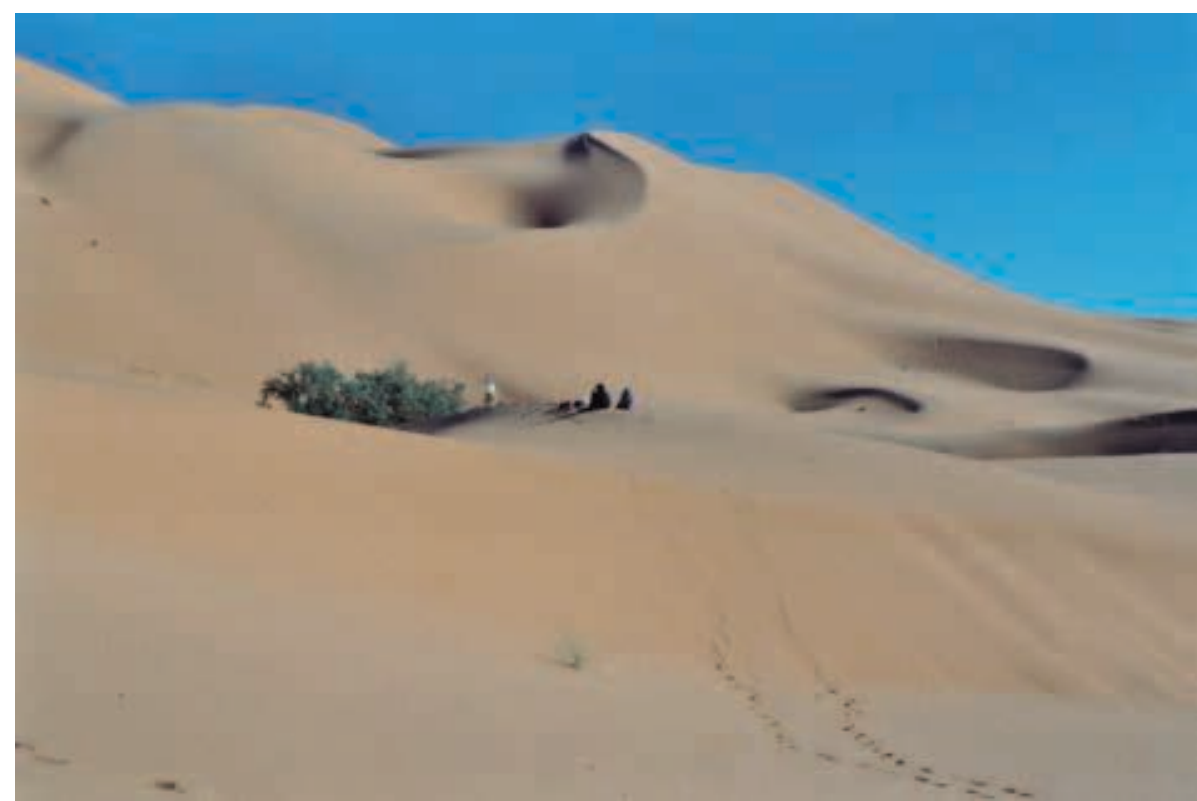

Bosquet de tamaris, lieu des voux adressés à Lala Merzouga

Les femmes ne montent pas au sommet, elles demeurent en contrebas, 2006 


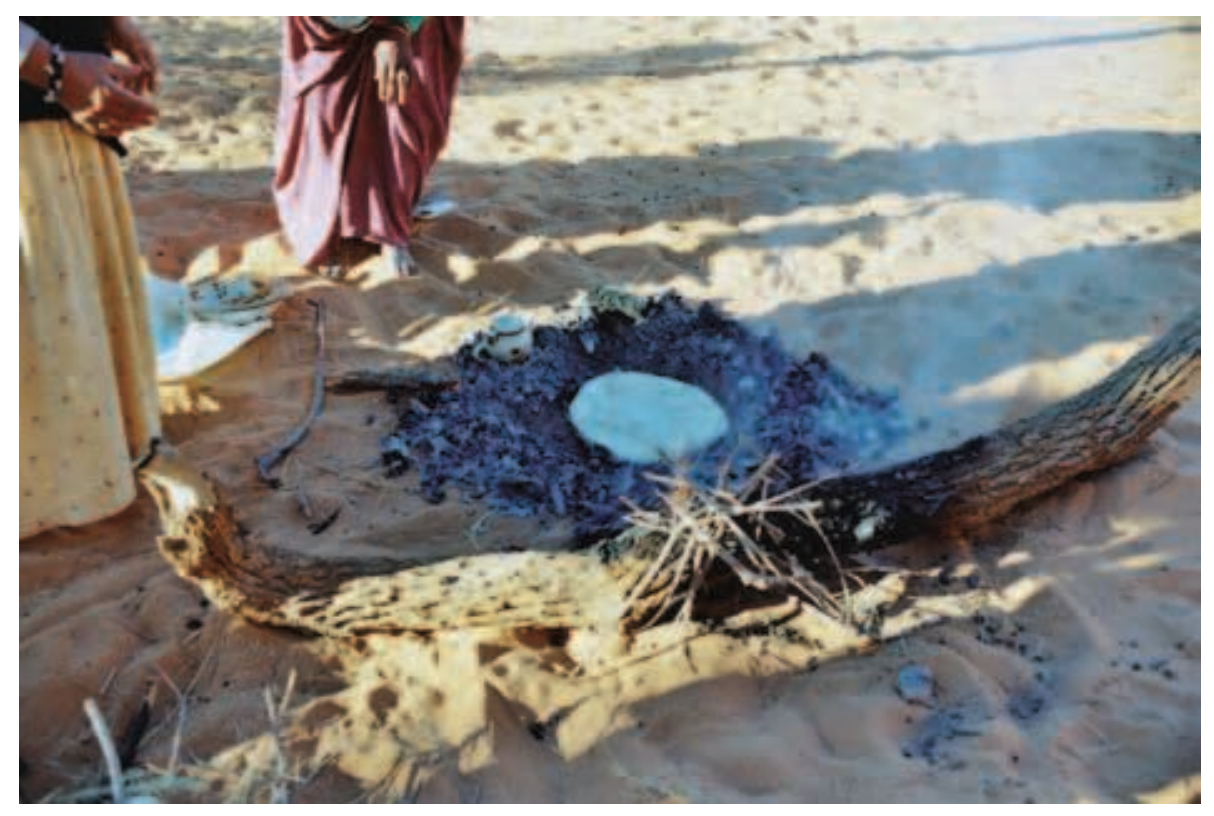

Dans ce bosquet sacré, il n'y a pas de circumambulations; une branche affaissée en forme d'arc de cercle sert parfois, selon les besoins, de lieu au rituel de franchissement accompagné du vœu murmuré et répété. Les femmes ramassent des branches de bois tombées au sol, mais évitent de prélever des branchages sur l'arbre. Elles allument un feu à même le sol, dans le sable. Du pain (aghrum n-aguensu) ${ }^{35}$ est cuit dans les braises et recouvert totalement de sable. C'est l'une des manières d'entrer directement en relation avec le sacré, en se nourrissant d'un mets ayant été en contact étroit avec le lieu et son substratum. L'immersion totale de la nourriture désormais chargée de la baraka de Lala Merzouga est très importante, c'est le contact et le toucher avec le sable qui est recherché. Une fois retiré du foyer, le pain est débarrassé des grains de sable en le frottant vigoureusement avec des feuilles de tamaris, ce qui lui donne une odeur et un goût bien spécifique, il est alors consommé par l'ensemble des femmes et des enfants présents.

Ce mode de cuisson était couramment usité lors des déplacements des nomades Ait Khebbach qui effectuaient des parcours transsahariens entre le Maroc et le Mali. Si la pratique de cette cuisson a décliné, les hommes vivant seuls optent encore en faveur de ce procédé. En effet, les fours à pain collectifs ${ }^{36}$ utilisés sont exclusivement féminins, aucun homme n'oserait y pénétrer et donc l'utiliser. Il y a aussi des raisons gustatives et sensorielles à l'utilisation et à la réactivation de ce procédé qui est désormais courant à l'occasion de retrouvailles familiales ou amicales. Il est alors nécessaire dans le cadre villageois de récupérer du sable de l'Erg et de le transporter dans la cour intérieure. Ce sont les vertus spécifiques du sable et la symbolique associée, mise en exergue du mode de vie nomade, qui sont recherchées dans cette technique de cuisson particulière.

Les discours tenus aux touristes occidentaux qui partent en bivouac au cœur de l'erg Chebbi à propos du pain cuit dans le sable sont différents et mettent en avant
Dépôt du pain sur les braises

Il est ensuite recouvert des mêmes braises et de sable pour quelques minutes de cuisson. 2006. 
Exemple d'utilisation du mode de cuisson dans le sable en milieu villageois

Le feu est entretenu afin qu'il y ait suffisamment de braises.

Le pain est agrémenté d'oignons, de carottes et de graisse.

Le pain est refermé sur son contenu.

Le foyer est aussi constitué de pierres elles-mêmes posées sur le sable pour une meilleure répartition de la chaleur.

Dépôt du pain sur les pierres chauffées.

Contrairement au pain cuit pour les touristes en bivouac, on utilise ici le fond d'une cuve en métal qui permet une cuisson à l'étouffée.

Le tout est recouvert de sable puis de braises.

2012
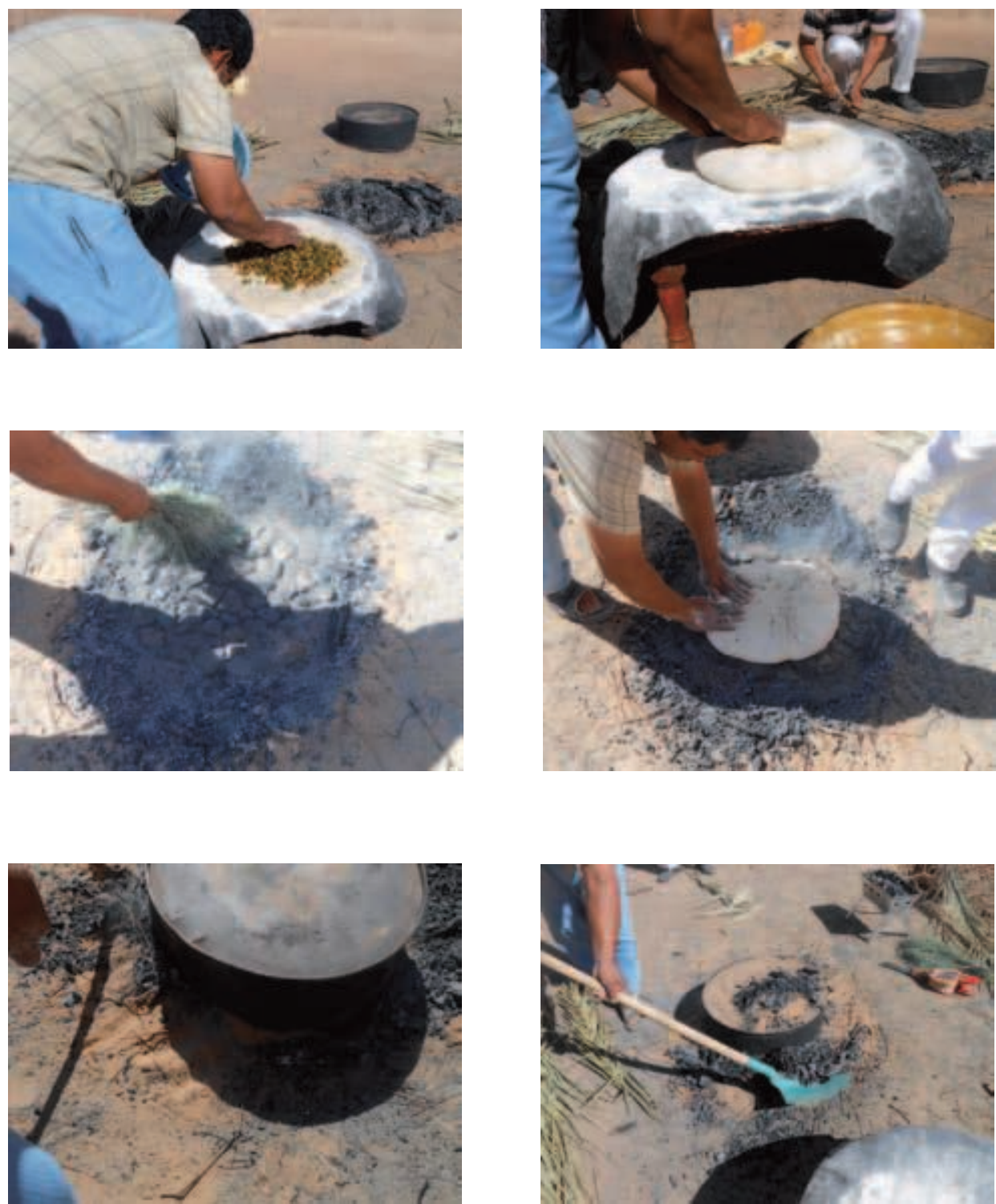
l'utilisation de l'environnement pour survivre. Ce sont principalement les chameliers qui les accompagnent et leur proposent de goûter ce pain. Il s'agit là aussi de «pain de l'intérieur ${ }^{37} »$ agrémenté de viande, d'oignons et d'épices. Cette technique fait désormais partie de tous les bivouacs proposés aux touristes, attisant l'admiration des voyageurs devant l'ingéniosité des nomades. La figure du nomade exerce une véritable fascination sur les touristes qui découvrent le désert, c'est aussi un moyen pour les Ait Khebbach de mettre en avant leur culture et leur ancien mode de vie.

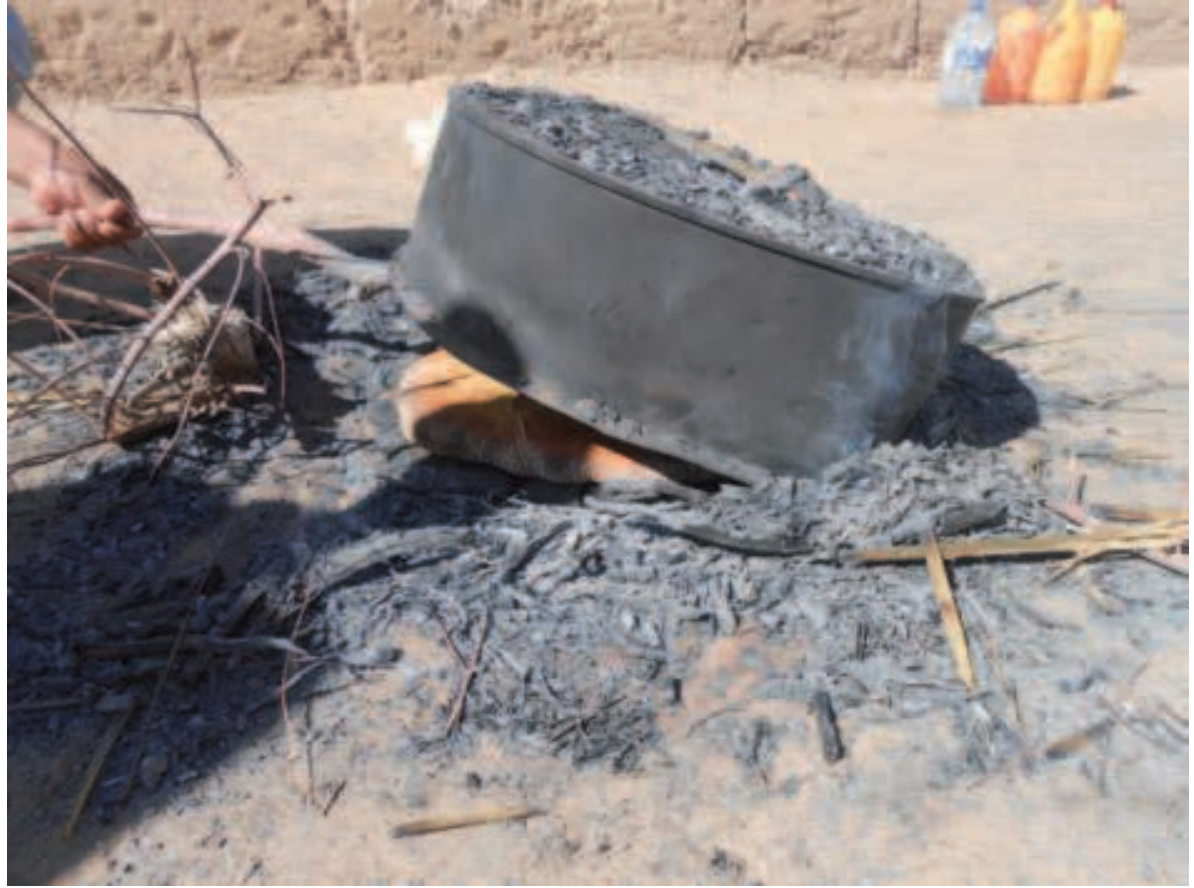

Sortie du pain

2012

\section{$\&$}

En conclusion, aujourd'hui en constante expansion, la pratique des « bains de sable » ne transfigure pas seulement les paysages sahariens par l'afflux notable de curistes durant l'été, elle permet aussi une réelle reconfiguration économique de ces espaces. Les touristes occidentaux viennent de moins en moins à la découverte du désert, la baisse de fréquentation est notable depuis quelques années alors que se pérennise celle d'un tourisme thérapeutique. Ces mobilités sont conséquentes, elles s'appuient sur une perception environnementale d'un monde urbain qui abîme et use les corps. On assiste aussi à une inversion, un retournement de l'image de l'autre. Le sable est au cour de ces représentations, il inverse en quelque sorte les anciennes hiérarchies comme celle entre urbanité et ruralité. Deux espaces qui au Maroc semblent très différents mais qui sont davantage en liens et en relations que ne le laisse penser une observation trop rapide. Les Marocains l'expriment fort bien « le monde rural est aux portes des villes ». Si l'espace saharien, du fait d'un isolement conséquent, a été longtemps considéré comme une zone inaccessible et peu attrayante pour les Marocains, les «bains de sable modifient cette image. Le contact s'établit entre des populations, un rapprochement s'opère et modifie de fait les a priori mais de là à transformer l'image du «bledard saharien »..

Louverture du désert à un tourisme thérapeutique national engendre des modifications notables et durables d'espaces; car malgré les perceptions négatives et parfois méprisantes des Sahariens envers les urbains et inversement, l'image de l'autre se modifie au miroir d'une altérité que la pratique des « bains de sable » impose. 


\section{NOTES}

Photo d'ouverture: Étendue de sable mais absence de dunes, à proximité du village de M'hammid, (C) M.-L. Gélard 2012. Sauf mention contraire, toutes les illustrations de l'article sont à mettre au crédit de l'auteur.

1. Le village de Merzouga, situé à l'extrême sud-est du Maroc, dans une enclave saharienne qui jouxte la frontière maroco-algérienne, naît dans les années 1950-1960 à la suite du processus de sédentarisation de la tribu des Ait Khebbach au milieu du xx $x^{e}$ siècle, il est le plus grand village de sédentarisation de la tribu. Celle-ci appartient à l'ensemble confédéré des Ait Atta qui occupe tout le Sud marocain, depuis le Haut Atlas jusqu'au Sahara et qui, à l'est, empiète sur l'actuel territoire algérien. C'est l'une des plus grandes confédérations de tribus du Maghreb, les Ait Khebbach en constituent la frange saharienne. Si certains nomadisent encore dans la région, les sécheresses endémiques jusqu'au début des années 2000 ont imposé la sédentarité.

2. L'erg Chebbi est la plus importante formation dunaire du Maroc, elle annonce le grand erg occidental algérien.

3. Je renvoie notamment aux analyses de V. Battesti (2009) à propos du tourisme oasien. D'autres textes sur le tourisme de désert, les imaginaires sousjacents ou les liens entre tourisme et développement se multiplient, citons par exemple V. Picon-Lefevbre et A. Chaouni (2012), mais rien sur les «bains de sable ». Les ornières du développement ont semblet-il occulté l'appréhension endogène de ces pratiques car si on lit parfois quelques données sur d'autres pratiques des touristes nationaux (quads et autres engins motorisés qui se multiplient depuis quelques années), c'est toujours dans la perspective du constat des dégâts sur les écosystèmes. À Merzouga, le tourisme est systématiquement analysé du point de vue de ses conséquences négatives (pollution, mauvaise gestion des déchets, constructions en zone inondable, sans autorisations, etc.) quand il n'est pas question de la fragilité des ressources du tourisme qui sont présentées comme transitoires (une transition qui dure depuis le début des années 1970). Citons enfin le livre de C. Cauvin-Verner (2007) qui propose une analyse du tourisme dans le Sud-Est marocain, mais qui ne fait nulle référence aux « bains de sable », pratique pourtant très développée, ce qui aurait pu permettre de contrecarrer, par la perspective comparative des tourismes nationaux et internationaux, les propos culturalistes qui émaillent l'ouvrage. Il n'y a donc point encore de littérature sur les « bains de sable », les seules données textuelles sont celles que l'on trouve dans la presse et les sites de voyages qui tous, eux, y font référence (j'y reviendrai). On notera une page qui lui est consacrée dans le bel ouvrage de A. Varichon et C. Roccella (2006: 44).

4. Un ouvrage pour enfants lui est consacré (Ouajjou: 2005).

5. Il est difficile de se référer à des données chiffrées précises mais à titre d'exemple, le village de Merzouga voit sa population plus que tripler durant la période des « bains de sable».

6. J'utiliserai dans la suite de ce texte, cette appellation pour désigner les amateurs et pratiquants des « bains de sable ». Il s'agit essentiellement de marocains arabophones habitant les grandes villes du royaume.

7. En ce qui concerne le Sud, c'est-à-dire le Tafilalt et Zagora.

8. En 2010, on comptait plus de 70 auberges entre le début de la formation dunaire au Nord et son extrémité sud soit environ $40 \mathrm{~km}$.

9. D'autres explications sont parfois avancées, j'ai entendu par exemple que le roi Hassan II serait l'inventeur de la pratique.

10. En effet, on ne retrouve pas cette seconde explication de manière itérative dans les discours, et elle semble une version spécifique au village de Merzouga, aussi il paraît peu probable que la prédiction thérapeutique ait été inventée par une seule personne.

11. Sur les valeurs identitaires féminines liées à la tente, je renvoie à M.-L. Gélard (2008).

12. L'eau sort sous forme de pus. Les blessures sont alors exposées au soleil.

13. Ces derniers viennent de l'agglomération de Rissani située à $35 \mathrm{~km}$ et réputée pour ses herboristes, fiqh (ces hommes érudits dans l'étude des textes religieux ont aussi des pouvoirs de guérison par l'utilisation et le recours à l'écrit coranique).

14. Depuis quelques années, certains les réalisent à la tombée du jour afin d'atténuer le choc thermique. Durant l'été 2014, on déplore des décès dus, explique-t-on à Merzouga, à l'absence de surveillance des curistes par leurs aides. En effet, certains aides s'occupent de plus de vingt curistes à la fois.

15. Concomitants à l'arrivée du tourisme dans les années 1970, de nouveaux usages du sable apparaissent 
comme la pratique du ski et aujourd'hui du surf. Le ski sur le sable est peu développé car il s'effectue sur sable mouillé ce qui est rare, le surf semble offrir un usage plus aisé mais peu développé.

16. La variabilité des pratiques est importante. Aujourd'hui, il semble que certains curistes puissent boire même pendant le bain de sable, dans ce cas on est plus proche des vertus d'un hammam sec et d'une circulation de la sueur, pensée comme un remède « dépuratif ».

17. Autrefois, la ville algérienne de Taghit faisait partie de l'aire de nomadisation des Ait Khebbach. La délimitation de la frontière par les autorités coloniales et la sédentarisation ont contraint les Ait Khebbach à un immobilisme qui les a aussi confinés dans l'enclave saharienne de Merzouga.

18. Pour plus de détails voir M.-L. Gélard (2014).

19. Terminologie usitée sur différent site: «Sablumthérapie, du latin sabulum = sable ». www.desert-amazigh.com. Notons le terme latin « arena » renvoyant aux jeux du cirque, espace couvert de sable.

20. Tout le monde peut s'improviser expert en «bains de sable».

21. La diminution du tourisme est notable, les responsables d'infrastructures hôtelières à Merzouga évoquent tous la «peur des touristes européens qui ne sont jamais venus au Maroc ».

22. Depuis quelques années, les terminologies officielles ont été établies par l'IRCAM, (Institut Royal de la Culture Amazigh) qui les diffuse. Le nom Amazigh, Imazighen au pluriel en langue tamazight signifie « Berbère(s) » et est utilisé désormais sous sa forme latinisée «Amazighes » (au lieu d'Imazighen). Ces terminologies ne sont pas anodines, elles témoignent d'un enjeu politique de taille (revendications identitaires). J'utilise pour ma part dans la majorité des cas les appellations employées par les Ait Khebbach eux-mêmes.

23. Comme on le sait, il existe plusieurs parlers berbères distincts au Maroc, celui du Rif (Tarifit), celui du Sud-Ouest (Cheulh) ou encore celui de l'Atlas et des confins sahariens (Tamazight).

24. J'ai présenté en janvier 2013 l'originalité de l'animal ainsi que la pratique des « bains de sable » à un journaliste de l'émission de télévision de France 3 « C'est pas sorcier » (J.-M. Sigot) dans le cadre d'une série de reportages sur le Sahara.

25. Les poissons de sable sont l'un des « jouets vivants » favoris des enfants, c'est ce que relève D. Champault à propos de l'oasis de Tabelbala situé à l'est de Merzouga, autrefois au cœur du parcours de nomadisation des Ait Khebbach. On brise les pattes de l'animal afin qu'il ne puisse s'échapper. Ils sont parfois traités comme de véritables poupées, « habillées de chiffons et dorlotées » (D. Champault 2003).
26. C'est une façon pour les habitants de Merzouga de retourner les moqueries qui sont le plus souvent celles des citadins à leur égard, souvent déconsidérés et regardés avec dédain. La figure du nomade est aussi l'objet d'une dévalorisation.

27. Le terme « auberge » continue d'être employé pour désigner des infrastructures hôtelières qui sont devenues de véritables hôtels comprenant jusqu'à 30 chambres, piscine, etc.

28. Propos recueillis à Hassylbyed, décembre 2011.

29. À l'époque, et encore aujourd'hui, il s'agit d'une pratique majoritairement masculine.

30. Militaire représentant de l'État dans les communes rurales.

31. L'urbanisation massive du Sahara est un phénomène bien documenté (voir notamment Belguidoum 2002, Pliez 2003, Kouzmine 2012).

32. En 2011, le Ramadan eut lieu du $1^{\text {er }}$ au 20 août, en 2010 du 11 août au 10 septembre et en 2009 du 22 août au 20 septembre.

33. 50 dh soit environ 5 euros. Le salaire moyen d'un ouvrier journalier est de 60 dh par jour.

34. Les couvertures sont vendues pour environ $30 \mathrm{dh}$ pièce. Certaines agences de voyages qui proposent des séjours «santé au désert » expliquent aux touristes/ curistes : «La couverture qui vous servira au sortir du bain de sable étant personnelle est détruite à votre départ elle vous sera facturée 20 euros».

35. Littéralement le "pain de l'intérieur » car on ajoute au cour du pain de la graisse, des oignons et des carottes, le tout finement râpé.

36. Le pain est cuit quotidiennement par les familles. L'usage des fours à pain est géré par un collectif féminin au gré des affinités parentales et électives et en fonction de la géographie des quartiers du village. Le four est allumé à tour de rôle en raison de la quantité de bois nécessaire, la combustion est ensuite maintenue par chacune des femmes venues cuire son pain.

37. Lorsque les touristes visitent la petite ville saharienne de Rissani, c'est le même type de pain qui leur est proposé, mais il est cette fois constitué de pain blanc (aghrum n-souk, littéralement le pain du marché), cuit à la boulangerie et appelé « pizza berbère ». Les touristes accompagnés de leur guide achètent la viande au boucher du souk puis elle est accommodée par des jeunes hommes, sortes de « cuisiniers » (oignons, épices, œufs durs, etc.) et apportée au boulanger pour la cuisson. Cette opération exige une organisation du travail et une division des rôles qui permet un bénéfice financier réparti entre plusieurs personnes. Là aussi les modes varient et avec eux les discours tenus aux touristes. 


\section{RÉFÉRENCES}

Battesti, V. 2009 Tourisme d'oasis. Les mirages naturels et culturels d'une rencontre?, Cahiers d'Études Africaines 193-194: 551-582.

Belguidoum, S. 2002 Urbanisation et urbanité au Sahara, Méditerranée (3/4) : 53-64.

Cauvin Verner, C. 2007 Au désert. Une anthropologie du tourisme dans le Sud marocain. Paris: L'Harmattan.

Champault, D. 2003 Les Jeux d'enfants dans l'oasis de Tabalbala, in Encyclopédie berbère, n 25 | Iseqqe-

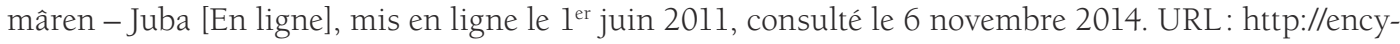
clopedieberbere.revues.org/1513.

Gélard, M.-L. 2014 Chaleur et dessiccation. Traitement du corps malade et esthétique, in M.-L. Gélard (dir.) Corps sensibles. Usages et langages des sens. Nancy: Presses Universitaires de Nancy, «Épistémologie du corps $»$.

— 2008 De la tente à la terre, de la terre au ciment... Persistance et permanence de la tente dans un village de sédentarisation (Merzouga, Maroc), Socio-Anthropologie 22: 123-143.

Kouzmine, Y. 2012 Le Sahara algérien. Intégration nationale et développement régional. Paris: L'Harmattan.

Ouajjou, S. 2005 Mi Lalla et Bassidi à Merzouga. Casablanca: Éditions La croisée des chemins.

Picon-Lefebvre, V. \& Chaouni, A. 2012 Desert tourism. Tracing the fragile edges of development. Cambridge: Harvard University.

Pliez, O. 2003 Villes du Sahara. Urbanisation et urbanité dans le Fezzan libyen. Paris: CNRS.

Varichon, A. \& Roccella, C. 2006 Ettre sable, Paris: Seuil.

Warnier, J.-P. 2009 Technology as efficacious action on objects... and subjects, Journal of Material Culture (14/4) : 459-470.

- 1999 Construire la culture matérielle. L’homme qui pensait avec ses doigts. Paris: PUF.

\section{POUR CITER CET ARTICLE}

Gélard, M.-L. 2014 Les « Bains de sable » dans le Tafilalt, in S. Boulay \& M.-L. Gélard, Vivre le sable! Corps, matière et sociétés, Techniques \& Culture 61: 100-121. 


\section{RÉSUMÉ}

Les « Bains de sable» dans le Tafilalt (Maroc). Au cœur de l'environnement des Sahariens, le sable et la chaleur sont instrumentalisés à des fins curatives. L'exemple le plus frappant est celui des « bains de sable » dont l'usage est en pleine expansion. Les douleurs articulaires et particulièrement les rhumatismes dont souffrent les urbains des principales villes du nord du Maroc au climat humide se soignent grâce à ces désormais célèbres « bains de sable ». Il s'agit de pratiques d'immersion dans le sable brûlant durant la période estivale pendant laquelle les températures sont très élevées. Le sable chaud $\left(70\right.$ à $\left.80^{\circ} \mathrm{C}\right)$ absorberait l'excédent d'humidité contenu dans le corps tout entier et plus particulièrement dans les os. Cette pratique se développe depuis quelques années et touche d'autres régions sahariennes marocaines, algérienne et égyptienne. L'engouement contemporain pour ces cures singulières transfigure les espaces sahariens par l'afflux démographique de ce tourisme thérapeutique. Ce texte propose de mettre en lumière les pratiques thérapeutiques et les discours curatifs qui se développent à propos des « bains de sable » tout en illustrant les modifications sociales sous-jacentes (représentation de l'autre, modifications de l'habitat, urbanisation massive, etc.). Une attention est aussi portée à l'utilisation du sable dans d'autres domaines comme celui de l'alimentation: cuisson du pain dans le sable. Sable, chaleur et dessiccation soulignent leur importance et leur instrumentalisation positive par les Sahariens, illustrant un environnement salvateur loin des préjugés habituels que véhicule l'hostilité du désert...

\section{ABSTRACT}

"Sand baths" in Tafilalt (Morocco). At the heart of the Saharan environment, sand and heat are instrumentalized for healing. The most striking example is "sand baths" whose use is expanding. Joint pain and rheumatism especially suffered by urban of major humid climate northern Morocco cities can be treated via the now famous "sand baths". It is practical immersion in burning sand during the summer when the temperatures are very high. The hot sand $\left(70\right.$ to $\left.80^{\circ} \mathrm{C}\right)$ absorb excess moisture content in the whole body, especially the bones. This practice has been developing for several years and affects other Saharan regions like Morocco, Algeria and Egypt. The contemporary infatuation for these peculiar cures transfigures Saharan spaces through the population influx of this health tourism. This text proposes to highlight the therapeutic practices and curative discourses that develop around "sand baths" while illustrating underlying social changes (representation of the other, habitat modification, massive urbanization, etc.). Attention is also focuse to the use of sand in other areas such as food: bread baking in the sand. Sand, heat and desiccation stress their importance and their positive instrumentalisation by Saharans, illustrating a salvation environment away from usual prejudices that convey hostility to desert...

\section{MOTS CLÉS}

«Bains de sable », Berbères, corps, tourisme thérapeutique, Sahara

\section{KEYWORDS}

"Sand baths", Berber, body, therapeutic tourism, Sahara 